\title{
Avaliação de um modelo experimental de enterocolite necrosante neonatal em ratos ${ }^{1}$
}

\author{
Evaluation of an experimental model of \\ necrotizing enterocolitis in rats
}

\begin{abstract}
Karine Furtado Meyer², José Luiz Martins ${ }^{3}$, Luiz Gonzaga de Freitas Filho ${ }^{4}$, Maria Luiza Vilela Oliva ${ }^{5}$, Francy Reis da Silva Patrício ${ }^{6}$, Maurício Macedo ${ }^{2}$, Lina Wang ${ }^{7}$

1. Trabalho realizado no Departamento de Cirurgia - Universidade Federal de São Paulo (UNIFESP) - Escola Paulista de Medicina (EPM). Brasil.

2. Aluno do Programa de Pós-Graduação em Cirurgia e Experimentação, nível de doutorado, UNIFESP-EPM. Brasil.

3. Professor Adjunto da Disciplina de Cirurgia Pediátrica. Livre Docente do Departamento de Cirurgia, UNIFESP-EPM. Brasil.

4. Professor Afiliado da Disciplina de Cirurgia Pediátrica do Departamento de Cirurgia, UNIFESP-EPM. Brasil.

5. Professor Adjunto da Disciplina de Biologia Molecular do Departamento de Bioquímica, UNIFESP-EPM. Brasil.

6. Professor Adjunto da Disciplina de Patologia Médica do Departamento de Patologia, UNIFESP-EPM. Brasil.

7. Médica Residente em Cirurgia Pediátrica- Hospital do Servidor Público Estadual de São Paulo. Brasil.
\end{abstract}

\section{RESUMO}

Objetivo: Avaliar um modelo experimental de enterocolite necrosante em ratos proposto por Okur e colaboradores em 1995. Métodos: Utilizou-se 28 ratos da raça EPM-Wistar no primeiro dia de vida, com peso entre 4 a 6 gramas. Os animais foram submetidos a hipóxia $(\mathrm{H})$ colocando os filhotes em uma câmara de gás $\mathrm{CO}_{2}$ para sacrifício de roedores onde receberam um fluxo de ar contendo $100 \%$ de $\mathrm{CO}_{2}$ durante 5 minutos. Após a hipóxia os animais foram reanimados (R) com fluxo de ar contendo $\mathrm{O}_{2}$ a 100\%, também durante 5 minutos. Os animais divididos em dois grupos: G1: controle ( $\mathrm{n}=12$ ): ratos não submetidos a H-R; G2: (n=16): ratos submetidos a H-R. Segmentos de intestino delgado e cólon foram preparados para análise histológica. O restante do intestino foi utilizado para dosagem de malondialdeído tecidual. Resultados: Dosagem de malondialdeído do G1 foi em média 1,05 (0,44-2,03) e do G2 foi em média 2,60 (0,59- 6,4) nmol MDA/mg proteína. O G2 teve média significativamente maior do que a do grupo controle $(p<0,002)$. Foi encontrada diferença estatisticamente significante entre os grupos de estudo quanto à distribuição do grau de lesão onde o grupo G1 apresentou graus significantemente menores do que o grupo G2. Conclusões: O modelo mostrou que a hipóxia neonatal em ratos provoca lesões na parede intestinal.. Apesar das lesões histológicas discretas é um bom método para avaliação da liberação de radicais livres teciduais.

Descritores: Enterocolite Necrosante. Modelos Animais. Ratos.

\begin{abstract}
Objective: To evaluate an experimental model of necrotizing enterocolitis in rats proposed by OKUR e col. in 1995. Methods: On their first day of life, 28 EPM-Wistar rats weighing between 4 and 6 grams were submitted to hypoxia (H) by placing them in a $\mathrm{CO}_{2}$ gas chamber for rodents' sacrifice, where they received a $100 \% \mathrm{CO}_{2}$ air flow for 5 minutes. After the hypoxia the animals were reanimated $(\mathrm{R})$ with a $100 \% \mathrm{O}_{2}$ air flow, also for 5 minutes. The animals were allocated in two groups: G1: control (n=12): rats not submitted to H-R; G2: $(n=16)$ : rats submitted to H-R. Segments of the small intestine and colon were prepared for histological analysis. The remaining intestine was used to measure tissular malondialdehyde. Results: Mean malondialdehyde dosages were 1.05 (0.44-2.03) and 2.60 (0.59- 6.4) nmol MDA/mg protein for G1 and G2, respectively. G2's mean value was significantly higher than in the control group ( $<<0.002)$. Significant statistical difference between the studied groups was found in relation the level of injury, with G1 presenting significantly lower levels than G2. Conclusions: The model showed that neonatal hypoxia may cause intestinal wall injury in rats. Despite the discreet histological injuries found, the method is suitable for evaluation of tissular free radicals.
\end{abstract}

Key words: Enterocolitis, Necrotizing. Models, Animal. Rats.

\section{Introdução}

Apesar dos avanços significativos na prática de cuidados neonatais, a enterocolite necrosante neonatal (ECN) continua sendo a mais importante causa de mortalidade e morbidade (20 a 35\%) em crianças com baixo peso ao nascer. A ECN é a emergência gastrointestinal mais comum nas unidades de cuidados intensivos neonatais; cerca de 5\% dos recém-nascidos (RN) com baixo peso ao nascer acabam desenvolvendo ECN ${ }^{1}$. A maior parte dos RN que desenvolvem ECN é tratada com nutrição parenteral e antibióticos de amplo espectro de ação, ficando a indicação operatória restrita às não infreqüentes complicações e tendo como principal objetivo atuar antes que ocorram perfurações 
intestinais, responsáveis pela péssima evolução destas crianças. Nestes casos, a mortalidade cirúrgica pode chegar a $45 \%^{2}$. As crianças que sobrevivem às ressecções de grandes extensões do intestino desenvolvem a conhecida síndrome do intestino curto, cuja morbidade acaba comprometendo de maneira definitiva o desenvolvimento pôndero-estatural, com implicações também no desenvolvimento neuro-psico-motor ${ }^{2}$. Diferentes hipóteses têm sido lançadas para explicar a origem da NEC, porém ela permanece como uma doença de etiologia desconhecida e de patogênese incerta. $O$ tratamento ainda é baseado em observações empíricas e nenhum método de prevenção mostrou sucesso absoluto ${ }^{1,2}$. A combinação de isquemia gastrointestinal, fruto de uma redistribuição do fluxo sangüíneo esplâncnico para órgãos vitais como coração e cérebro, alimentação enteral, possivelmente predispondo à lesão da camada mucosa e mais a translocação de bactérias patogênicas, tudo isto associado a uma imaturidade dos mecanismos imunitários intestinais, estariam envolvidos em seu desenvolvimento ${ }^{1,2}$. Muitos modelos animais foram usados para avaliar os efeitos da hipóxia na etiopatogenia da ECN e demonstraram que o decréscimo da perfusão intestinal e que a lesão tecidual seriam causadas por um mecanismo de isquemia e reperfusão ${ }^{3-12}$. O objetivo de nosso estudo é avaliar um modelo experimental de ECN em ratos proposto por em 1995 por Okur e col. ${ }^{9}$.

\section{Métodos}

O experimento foi aprovado pelo Comitê de Ética em Pesquisa da Universidade Federal de São Paulo- UNIFESPEPM, com o protocolo n ${ }^{\circ}$ 5660/04.

\section{Amostra}

Foram utilizados 28 ratos recém-nascidos da linhagem OUT B EPM-1 Wistar (Rattus norvegicus albinus, Rodentia mamalia), com peso variando de 4 a 6 gramas.

- G 1: $(N=12)$ : ratos que não foram submetidos a hipóxia-reoxigenação.

- G 2: $(\mathrm{N}=16)$ : ratos que foram submetidos somente a hipóxia-reoxigenação.

\section{Experimento}

Foi utilizado o modelo de ECN descrito por Okur e col. ${ }^{9}$ já reproduzido por outros autores ${ }^{10,12}$. Os animais foram submetidos a hipóxia em uma câmara (Câmara de Gás $\mathrm{CO}_{2}$, para sacrifício de roedores, modelo CGSCO2G - Marca Beiramar), onde receberam um fluxo de ar contendo 100\% de $\mathrm{CO}_{2}$ durante 5 minutos. Após a hipóxia os animais foram reanimados com fluxo de ar contendo $\mathrm{O}_{2}$ a $100 \%$ durante 5 minutos e em seguida foram mantidos junto às respectivas mães em ambiente normotérmico. Todos os animais receberam leite materno antes e após o procedimento. Todos os animais foram submetidos à eutanásia por decapitação no quarto dia de vida. Segmentos de intestino delgado e cólon, cada um com $1 \mathrm{~cm}$ de extensão, foram preparados para análise histológica. $\mathrm{O}$ restante do intestino foi então removido em bloco e imediatamente congelado a $-80^{\circ} \mathrm{C}$ para posterior homogeneização e dosagem de malondialdeído tecidual.

\section{Exame histopatológico}

Os fragmentos de $1 \mathrm{~cm}$ foram fixados em formol a $10 \%$, desidratados, embebidos em parafina e corados com hematoxilina-eosina. Foram realizados cortes de 6 micrômetros em cada fragmento e as lâminas serão analisadas à microscopia óptica pelo patologista, sem o prévio conhecimento sobre o grupo pertencente de cada rato, e foram classificados de acordo com o grau de lesão tecidual segundo Chiu e col. ${ }^{13}$ :

- Grau 0: mucosa sem alterações.

- Grau 1: vilosidades bem constituídas, sem lise celular ou processo inflamatório, porém, com formação do espaço subepitelial de Grunhagen.

- Grau 2: presença de lises celulares, formação do espaço subepitelial de Grunhagen e espaçamento aumentado entre as vilosidades.

- Grau 3: destruição da porção livre das vilosidades, presença de capilares dilatados e de células inflamatórias.

- Grau 4: destruição estrutural das vilosidades, havendo apenas esboço de algumas, formadas por células inflamatórias e material necrótico, com hemorragia e ulceração glandular basal.

- Grau 5: destruição de toda túnica mucosa, não mais sendo observado qualquer estrutura glandular, mas apenas material amorfo depositado sobre a tela submucosa.

\section{Determinação de malondialdeído tecidual}

Malondialdeído (MDA) é um produto final da peroxidação de lipídeos e é um parâmetro bem estabelecido para determinar o aumento de radicais livres no tecido intestinal. Para determinar os níveis de MDA será utilizado o método do ácido tiobarbitúrico (TBA), originalmente proposto por Kohn e Liversedge ${ }^{14}$ e os valores serão expressos como nanomoles de MDA por miligrama de proteína (nmol MDA/mg proteína) e por grama de tecido. $\mathrm{O}$ conteúdo protéico do homogenado será determinado pelo procedimento de "coomassie brilhant blue" (CBB). As amostras teciduais foram descongeladas, anotou-se o peso e adicionou-se 5 vezes o peso em gramas de solução tampão de TRIS $0,01 \mathrm{M} / \mathrm{pH} 7,4$. As amostras teciduais foram homogeneizadas, 4 séries de 30 segundos em banho de gelo e posteriormente, centrifugadas por 5 minutos a 10.000 rpm a $4^{\circ} \mathrm{C}$.

\section{Dosagem de proteína}

O reagente CBB interage com a proteína, permitindo a quantificação da mesma utilizando uma curva padrão de albumina com concentrações conhecidas.

Preparo do reagente CBB: dissolveu-se $100 \mathrm{mg}$ de CBB 250G em $50 \mathrm{ml}$ de etanol 95\% (agitou-se bem até dissolver). Adicionou-se $100 \mathrm{ml}$ de ácido fosfórico 85\%, com agitação constante. Acrescentou-se água destilada para um volume final de 1 litro. O reagente foi deixado em repouso por 24 horas e em seguida, filtrado e mantido em frasco escuro.

Utilizando uma solução estoque de albumina bovina (BSA) $10 \mathrm{mg} / \mathrm{ml}$, preparou-se $200 \mathrm{ul}$ das seguintes soluções: 0,1 mg/ml, 0,2 mg/ml, 0,4 mg/ml, 0,8 mg/ml e $1 \mathrm{mg} / \mathrm{ml}$ (albumina bovina/ml de água) para fazer a curva padrão de albumina 
bovina (curva padrão para dosagem de proteínas). Colheram-se $25 u$ l e $50 u$ l do homegenato, posteriormente diluídos em 5 vezes em tampão de TRIS 0,01M/pH 7,4; acrescentou-se $2,5 \mathrm{ml}$ do CBB e foram feitas as leituras em $595 \mathrm{~nm}$ após 10 min da adição do reagente de CBB.

\section{Dosagem de malondialdeído}

Coletou-se 400 ìL do sobrenadante do homogenato centrifugado e a ele foram adicionados: $1 \mathrm{~mL}$ de ácido tricloroacético $20 \%$ e 400 ìL de ácido tiobarbitúrico a 1,6\% sendo essa mistura incubada por 30 minutos a $95^{\circ}$ Celsius. Os lípideos foram extraídos pela adição de n-butanol (1,6 $\mathrm{mL}$ ) e vigorosa agitação. A amostra foi centrifugada novamente por 10 minutos a 3000 rpm. A absorbância de camada orgânica foi determinada pela absorção em 532, 510 e $560 \mathrm{~nm}$.Foi utilizada a seguinte equação proposta para minimizar a interferência dos pigmentos heme e da hemoglobina na dosagem de $\mathrm{MDA}^{15}$ :

$$
\operatorname{MDA}_{532}=1,22\left[\left(A_{532}\right)-(0,56)\left(A_{510}\right)+(0,44)\left(A_{560}\right)\right]
$$

A curva de calibração foi traçada com 1,3,3 tetrametoxipropano malondialdeído bis (acetildimetilo).

A concentração de malondialdeído foi expressa em nanomoles de malondialdeído por grama de tecido e em nanomoles de malondialdeído por miligrama de proteína.

\section{Estudo estatístico}

As variáveis quantitativas contínuas foram representadas por média, erro padrão da média (ep), valores mínimo e máximo e as discretas por freqüência absoluta (n) e relativa (\%). O teste de Kolmogorov-Smirnov foi aplicado para testar a presença de distribuição normal nas variáveis quantitativas dentro de cada grupo de estudo. Na comparação entre os grupos de estudo em relação às médias das variáveis nmol/mg de proteína foi utilizada a técnica de Análise de Variância (ANOVA) com o fator fixo Grupo. As diferenças foram localizadas pelo teste de comparações múltiplas de Tukey. Foi aplicada a Prova não paramétrica de Kruskal-Wallis para amostras independentes na análise do grau de lesão tecidual nas 3 localizações avaliadas. As diferenças foram localizadas pelo teste de comparações múltiplas de Dunn. Adotou-se o nível de significância de $0,05(\mathrm{a}=5 \%$ ) e níveis descritivos (p) inferiores a esse valor foram considerados significantes e representados por *.

\section{Resultados}

\section{Dosagem de malondialdeído tecidual (Tabela 1)}

Foi encontrada diferença estatisticamente significante entre os grupos de estudo quanto à média de nmol de MDA/ mg de proteína nos animais $(p=0,002)$ e as comparações entre os pares de grupos mostraram que:

- o grupo G2 mostrou média significantemente maior do que a do grupo G1 ( $p=0,015)$.
TABELA 1 - Dosagem de MDA tecidual

\begin{tabular}{llllll}
\hline & \multicolumn{5}{c}{ nmol/mg de Proteína } \\
\cline { 2 - 6 } Grupo & Média & e.p. & Mínimo & Máximo & n \\
\hline G1 & 1,0539 & 0,1419 & 0,4455 & 2,0337 & 12 \\
G2 & 2,6007 & 0,4677 & 0,5993 & 6,4034 & 16 \\
\hline
\end{tabular}

Análise de Variância: $\mathrm{p}=0,002$ *

Análise histológica (Figuras 1-6)
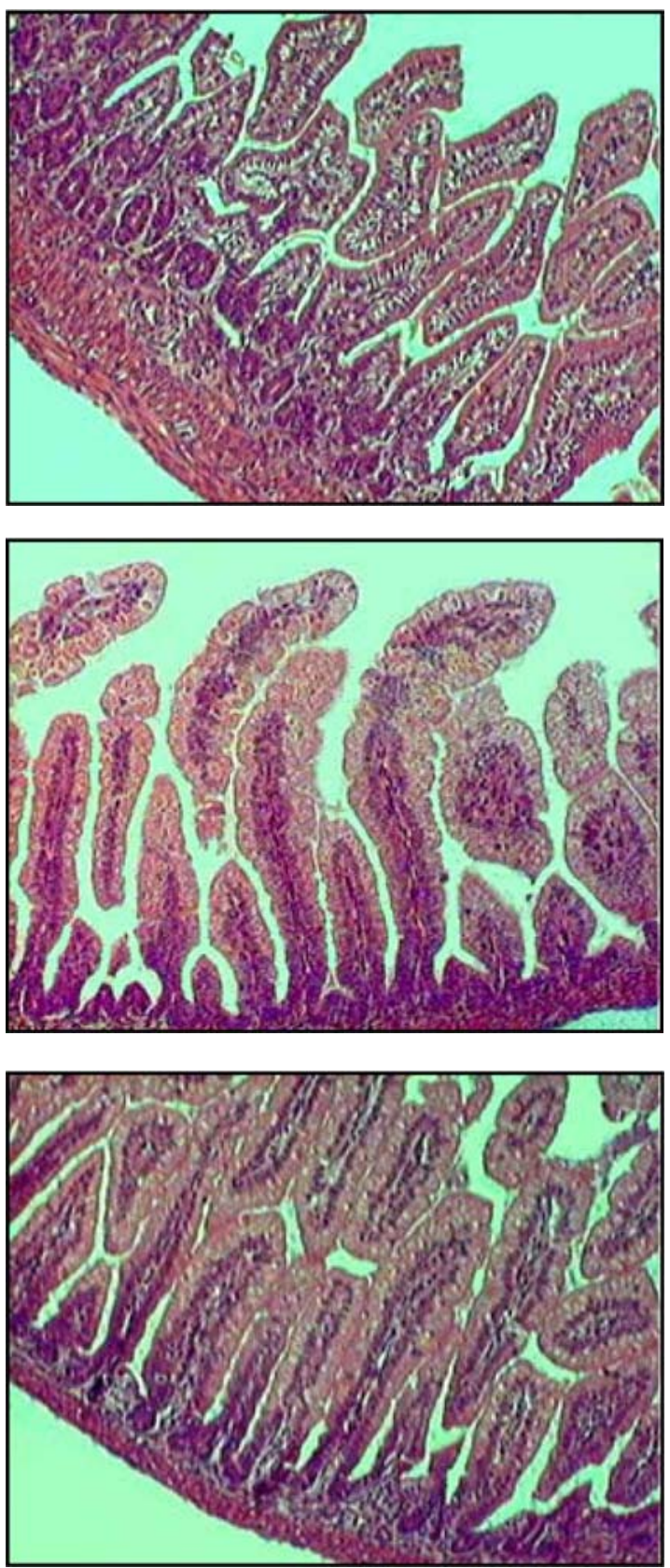

FIGURA 1 - Fotomicrografia de corte histológico de intestino do animal do Grupo Controle mostrando mucosa sem alterações (grau 0). 

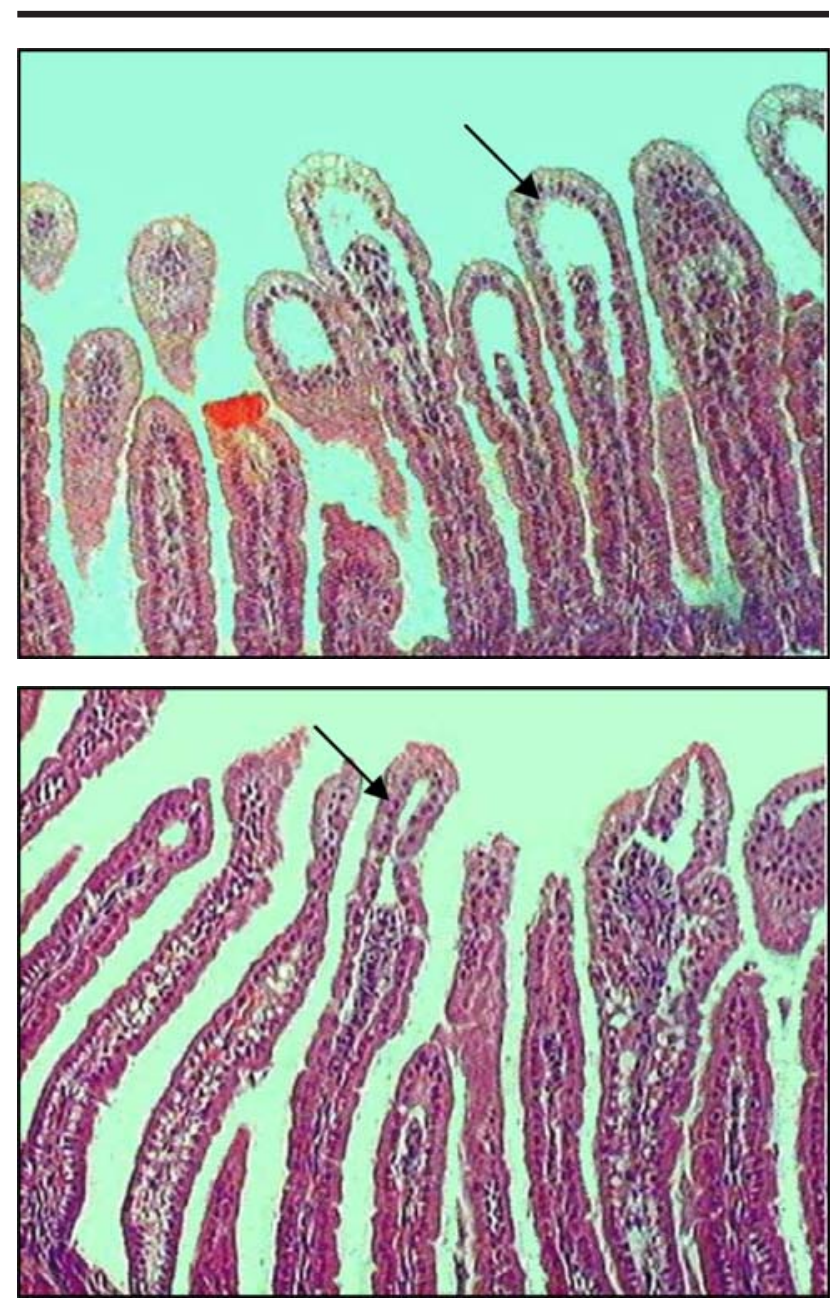

FIGURA 2 - Fotomicrografia de corte histológico de intestino do animal do Grupo Controle mostrando mucosa com vilosidades bem constituídas, sem lise celular ou processo infamatório, porém, com formação do espaço subepitelial de Grunhagen- seta preta (grau 1).

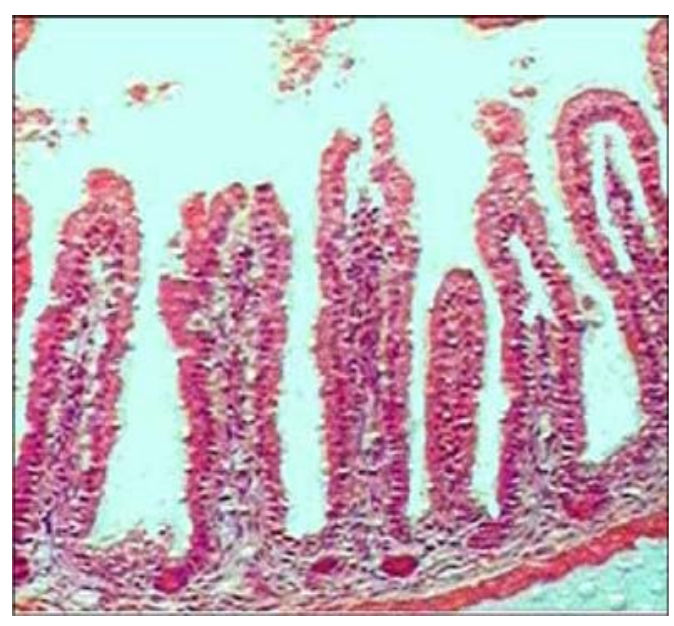

FIGURA 3 - Fotomicrografia de corte histológico de intestino do animal do Grupo H-R mostrando mucosa com presença de lises celulares, formação do espaço subepitelial de Grunhagen e espaçamento aumentado entre as vilosidades (grau 2).
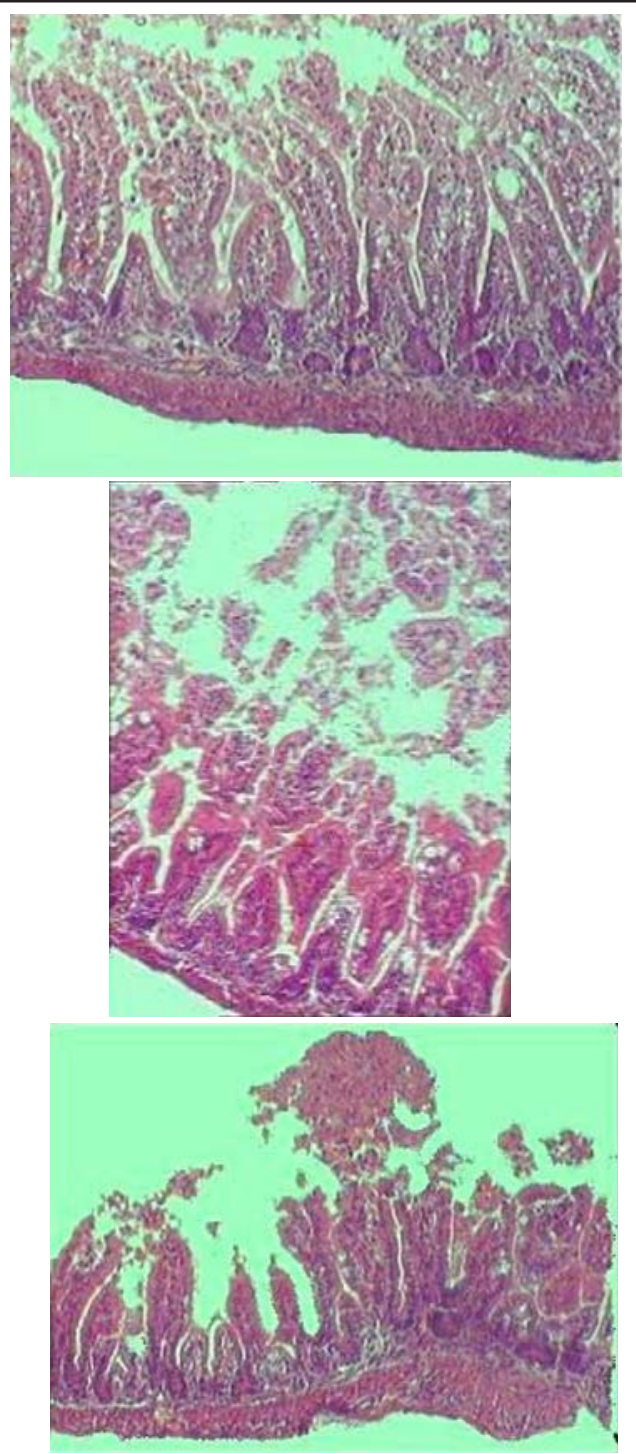

FIGURA 4 - Fotomicrografia de corte histológico de intestino do animal do Grupo H-R mostrando mucosa com destruição da porção livre das vilosidades, presença de capilares dilatados e de células inflamatórias (grau 3).

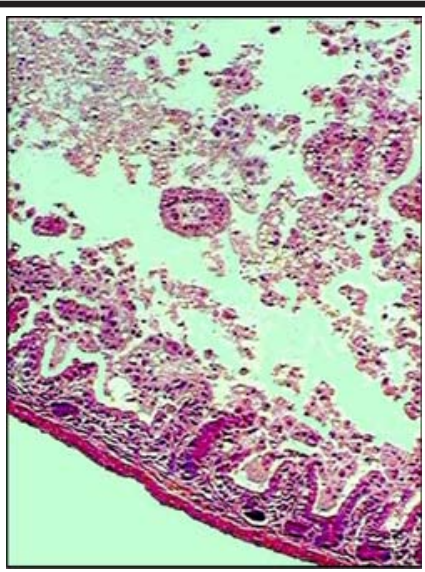

FIGURA 5 - Fotomicrografia de corte histológico de intestino do animal do Grupo H-R mostrando mucosa com destruição estrutural das vilosidades, havendo apenas esboço de algumas, formadas por células inflamatórias e material necrótico, com hemorragia e ulceração glandular basal (grau 4). 


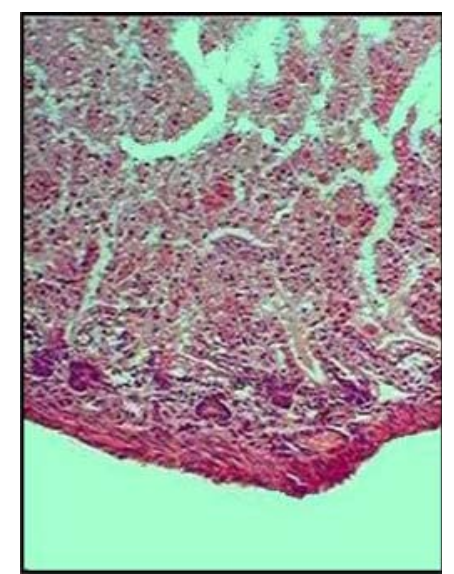

FIGURA 6 - Fotomicrografia de corte histológico de intestino do animal do Grupo H-R mostrando mucosa com destruição de toda túnica mucosa, não sendo mais observado qualquer estrutura glandular, mas apenas material amorfo depositado sobre a tela submucosa (grau 5).

Grau de lesão tecidual no intestino delgado (Tabela 2)

Foi encontrada diferença estatisticamente significante entre os grupos de estudo quanto à distribuição do grau de lesão tecidual no intestino delgado dos animais ( $p<0,001)$, onde o grupo G1 apresentou graus significantemente menores do que os dos grupos G2.

TABELA 2 - Análise histológica no intestino delgado

\begin{tabular}{llllll}
\hline \multirow{2}{*}{ Grau } & \multicolumn{2}{c}{$\begin{array}{c}\text { Controle } \\
(\mathrm{n}=12)\end{array}$} & & \multicolumn{2}{c}{$\begin{array}{c}\mathrm{H} / \mathrm{O} \\
(\mathrm{n}=16)\end{array}$} \\
\cline { 2 - 3 } \cline { 6 - 7 } & $\mathrm{N}$ & Escore & & $\mathrm{N}$ & Escore \\
\hline 0 & 10 & 83,3 & & 1 & 6,3 \\
1 & 1 & 8,3 & & 1 & 6,3 \\
2 & 0 & 0,0 & & 3 & 18,8 \\
3 & 1 & 8,3 & & 7 & 43,8 \\
4 & 0 & 0,0 & & 3 & 18,8 \\
5 & 0 & 0,0 & & 1 & 6,3 \\
\hline
\end{tabular}

\section{Grau de lesão tecidual no cólon (Tabela 3)}

Foi encontrada diferença estatisticamente significante entre os grupos de estudo quanto à distribuição do grau de lesão tecidual no cólon dos animais ( $\mathrm{p}=0,003)$, onde o grupo $\mathrm{G} 1$ apresentou graus significantemente menores do que os dos grupos G2.

TABELA 3 - Análise histológica no cólon

\begin{tabular}{llllll}
\hline \multirow{2}{*}{ Grau } & \multicolumn{2}{c}{$\begin{array}{c}\text { Controle } \\
(\mathrm{n}=12)\end{array}$} & & \multicolumn{2}{c}{$\begin{array}{c}\mathrm{H} / \mathrm{O} \\
(\mathrm{n}=16)\end{array}$} \\
\cline { 2 - 3 } \cline { 6 - 7 } & $\mathrm{N}$ & Escore & & $\mathrm{N}$ & Escore \\
\hline 0 & 10 & 83,3 & & 2 & 12,5 \\
1 & 0 & 0,0 & & 2 & 12,5 \\
2 & 1 & 8,3 & & 8 & 50,0 \\
3 & 1 & 8,3 & & 0 & 0,0 \\
4 & 0 & 0,0 & & 2 & 12,5 \\
5 & 0 & 0,0 & & 2 & 12,5 \\
\hline
\end{tabular}

\section{Discussão}

Vários modelos animais foram propostos para demonstrar a importância da hipóxia no desenvolvimento das lesões na ECN ${ }^{3-12}$. Barlow e Santulli ${ }^{3,5}$ provocaram lesão intestinal em ratos recém-nascidos alimentados com leite artificial, colocando os animais em saco plástico durante 3 a 5 minutos por dia. Harrison e col. ${ }^{4}$ demonstraram alterações precoces na mucosa intestinal e nas células capilares endoteliais pela microscopia eletrônica em cães recémnascidos submetidos a hipóxia com $7 \%$ de $\mathrm{O}_{2}$ por duas horas. Hansbrough e col. ${ }^{6}$, utilizando cães recém-nascidos, provocaram necrose isquêmica intestinal ao produzir hipóxia com $\mathrm{O}_{2}$.a $10 \%$ por 2 horas. Cohen e col. ${ }^{7}$ submeteram porcos recém-nascidos a uma pressão parcial de $\mathrm{O}_{2}$ de $50 \%$, conseguindo obter evidências microscópicas e macroscópicas de ECN. Caplan e col. ${ }^{8}$ descreveram um modelo de ECN alimentando os animais com fórmula artificial e submetendo-os a 60 segundos de asfixia com 100\% de nitrogênio duas vezes ao dia seguido por exposição ao frio.

Okur e col. ${ }^{9}$ demonstraram lesão de mucosa em ratos recém-nascidos submetidos a 5 minutos de hipóxia (100\% de dióxido de carbono) e 5 minutos de reoxigenação (100\% de oxigênio). Outros pesquisadores conseguiram também demonstrar a lesão isquêmica da mucosa intestinal neste modelo igual ao utilizado neste trabalho ${ }^{10,12}$.

Uma das razões aceita para explicar a lesão provocada pela hipóxia, seria que a asfixia neonatal levaria a uma redistribuição do fluxo sanguíneo ao provocar uma vasoconstrição esplâncnica, desviando o fluxo para órgãos vitais como coração e cérebro e provocando uma isquemia intestinal $^{5}$. Vários mecanismos estão envolvidos no início e progressão desta lesão por isquemia, como: o aumento da produção de peróxidos hiper reagentes, aumento da síntese de moléculas de adesão com infiltração de neutrófilos, aumento da peroxidação lipídica e a produção aumentada de mediadores inflamatórios como as citocinas ${ }^{25}$.

A peroxidação lipídica é um processo complexo que pode ocorrer em membranas biológicas constituídas de ácidos graxos poliinsaturados reagentes ao oxigênio molecular, levando à produção de hidroperóxidos lipídicos e seus metabólitos. A maioria dos casos envolvendo peroxidação lipídica inicia-se através de uma reação em cadeia que se propaga, mediada pela presença de radicais livres. Os hidroperóxidos lipídicos acumulam-se na membrana, inativando seus receptores e enzimas, prejudicando suas funções, levando a sua desestabilização e tornando-a permeável a íons. Um método simples e de alta sensibilidade muito utilizado como um marcador de peroxidação lipídica é a reação de substâncias reativas ao ácido tiobarbitúrico, dentre elas os derivados dos hidroperóxidos lipídicos, como o malondialdeído, MDA. O MDA é o melhor indicador de peroxidação lipídica provocada por radicais livres ${ }^{10}$. No modelo estudado os ratos submetidos a hipóxia-reoxigenação tiveram valores de MDA significativamente maiores que o grupo controle demonstrando a maior liberação de radicais livres intestinal nos ratos submetidos a hipóxia.

O modelo utilizado no experimento mostrou-se útil e de fácil reprodução. Uma de suas vantagens é não necessitar da administração de fórmulas enterais para obtenção de 
lesão intestinal. Uma importante limitação, que já havia sido demonstrada anteriormente ${ }^{10}$, no entanto, foi a discreta alteração histológica que se obteve nos diferentes grupos. Özkan e col. ${ }^{29}$ propuseram, ao utilizar o mesmo modelo, submeter os animais a repetidos períodos de hipóxiareoxigenação, capazes de causar lesões intestinais mais pronunciadas.

Outra limitação para este modelo é o pequeno tamanho dos animais. Os ratos no primeiro dia de vida pesam entre 4 e 6 gramas o que dificulta o estudo de drogas administradas por via oral e impede o estudo de drogas utilizadas por via endovenosa. Também, nos animais deste tamanho, não é possível o estudo de métodos de tratamento.

\section{Conclusões}

- O modelo mostrou que a hipóxia neonatal em ratos provoca lesões na parede intestinal.

- Apesar das lesões histológicas discretas é um bom procedimento para avaliação da liberação de radicais livres teciduais.

\section{Referências}

1. Kliegman RM, Walker A, Yolken RH. Necrotizing enterocolitis. Research agenda for a disease of unknown etiology and pathogenesis. Clin Perinatol. 1994;21:437-55.

2. Ladd AP, Rescorla FJ, West KW, Scherer LR, Engum SA, Grosfeld JL. Long-term follow-up after bowel ressection for necrotizing enterocolitis: factors affeting outcome. J Pediatr Surg. 1998;33:967-72.

3. Barlow W, Santulli TV, Heird WC, Pitt J, Blanc WA, Schullinger JN. An experimental study of acute neonatal enterocolitis: the importance of breast milk. J Pediatr Surg.1974;9(5):587-94.

4. Harrison MW, Connell RS, Campbell JR, Webb MC. Microcirculatory changes in gastrointestinal tract of the hypoxic puppy: an electron microscope study. J Pediatr Surg. 1975;10:599-608.

5. Barlow B, Santulli TV. Importance of multiples episodes of hipoxia or cold stress on the development of enterocolitis in an animal model. Surgery. 1975;77:687-90.
6. Hansbrough F, Priebe CJ, Falterman KW, Bornside GH, Welsh RA. Pathogenesis of early necrotizing enterocolitis in the hypoxic neonatal dog. Am J Surg. 1983;145:169-75.

7. Cohen IT, Nelson SD, Moxley R, Hirsh MP, Couihan TC, Martin RF. Necrotizing enterocolitis in a neonatal piglet model. J Pediatr Surg. 1991;26:598-601.

8. Caplan MS, Hedlund E, Adler L, Hsueh W. Role of asphyxia and feeding in a neonatal rat model of necroting enterocolitis. Pediatr Pathol Lab Med. 1994;14:1017-28.

9. Okur H, Küçükaydin M, Köse K, Kontas O, Dogan P, Kazez A. Hipoxia-induced necrotizing enterocolitis in the immature rat: the role of lipid peroxidation and management by vitamin E. J Pediatr Surg. 1995;30:1416-9.

10.Kazez A, Küçükaydin N, Küçükaydin M, Kontas O, Okur H, Dogan P. A model of hipoxia-induced necrotizing enterocolitis: the role of distension. J Pediatr Surg. 1997;32:1466-9.

11.Caplan M, Miller-Catchpole R, Kaup S, Russel T, Lickerman M, Amer M, Xiao Y, Thomson R Jr. Bifidobacterial supplementation reduces the incidence of necrotizing enterocolitis in a neonatal rat model. Gastroenterology. 1999;117:577-83.

12.Östürk H, Dokucu AI, Ögun C, Büyükbayram H. Protective effects of recombinant human interleukin-10 on intestines of hipoxia-induced necrotizing enterocolitis in immature rats. J Pediatr Surg. 2002;37:1330-3.

13. Chiu CJ, McArdle AH, Brown R, Scott HJ, Gurd FN. Intestinal mucosal lesion in low-flow states. Arch Surg. 1970; 101: 478-83.

14.Kohn HI, Liversedge M. On a new aerobic metabolite whose production by brain is inhibited by apomorphine, emetine, ergotamine, epinephrine and manadione. J Pharmacol Experimen Ther. 1944;82:292-300.

15.Pyles LA, Stejskal EJ, Einzig S. Spectrophotometric measurement of plasma 2-thiobarbituric acid-reactive substances in the presence of hemoglobin and bilirubin interference. Proc Soc Exp Biol Med. 1993;202:407-19.

16.Tardini MAS, Yoshida WB: Lesões cerebrais decorrentes de isquemia e reperfusão na cirurgia de endarterectomia de carótida. J Vasc Br. 2003;2:119-28.

\section{Correspondência:}

Karine Furtado Meyer.

Rua Rio Grande, 551/162 ${ }^{a}$

04018-001 São Paulo - SP Brasil

Tel/Fax: (11)5084-5342

karine meyer@uol.com.br
Conflito de interesse: nenhum Fonte de financiamento: FAPESP

(processo ${ }^{\circ}$ 2004/12449-3)

Recebimento: 26/10/2005

Revisão: 23/11/2005

Aprovação: 20/12/2005

\section{Como citar este artigo:}

Meyer KF, Martins JL, Freitas Filho LG, Oliva MLV, Patrício FRS, Macedo M, Wang L. Avaliação de um modelo experimental de enterocolite necrosante neonatal em ratos. Acta Cir Bras. [periódico na Internet] 2006 Mar-Abr;21(2). Disponível em URL: http://www.scielo.br/acb 